PACS 61.48.Gh, 68.37.Ps, 68.65.Pq, 78.30.-j, 78.67.Wj, 81.05.ue

\title{
The growth of weakly coupled graphene sheets from silicon carbide powder
}

\author{
V.S. Kiselov, P.M. Lytvyn, A.S. Nikolenko, V.V. Strelchuk, Yu.Yu. Stubrov, M. Tryus and A.E. Belyaev \\ V. Lashkaryov Institute of Semiconductor Physics, NAS of Ukraine, \\ 41, prospect Nauky, 03028 Kyiv, Ukraine
}

\begin{abstract}
A simple method for production of weakly coupled graphene layers by hightemperature sublimation of polycrystalline $\mathrm{SiC}$ is presented. The method allows manufacturing carbon-based composite with a high content of weakly coupled graphene layers in large-scale production. The study of the obtained carbon-based material by means of scanning electron microscopy, Raman spectroscopy and atomic force microscopy detected graphene plates with lateral size of up to tens of micrometers. The obtained graphene sheets are shown to have very high crystal perfection, low concentration of defects and weak interlayer coupling, which depends on the growth conditions. The proposed method of producing graphene-based composites is supposed to be very promising due to its relative simplicity and high output.
\end{abstract}

Keywords: graphene sheets, thermal decomposition, $\mathrm{SiC}$, atomic force microscopy, micro-Raman spectroscopy.

Manuscript received 08.04.14; revised version received 18.08.14; accepted for publication 16.09.14; published online 30.09.14.

\section{Introduction}

A single monolayer of graphite, widely known as graphene, has recently attracted considerable interest due to the set of its unique properties. It shows a novel magneto- transport properties [1-3], high carrier mobility and ballistic transport up to room temperature [4]. Graphene should be used in ultra-sensitive gas sensors [5], in spintronics [6,7], in a terahertz oscillators [8] and in variety of other promising applications including development of silicon electronics alternatives [9]. Requirements to graphene properties vary according to the specific application. Some applications such as transparent electrodes and sensors need thin films of graphene, but another one, like to energy storage devices and polymer composites require relatively large quantities of graphene nanosheets or plates [10].
Synthesis of monolayer graphite is known since 1975, when B. Lang et al. [11] shown formation of mono- and multilayered graphite by thermal decomposition of carbon on monocrystalline $\mathrm{Pt}$ substrates. However, the process was not studied extensively at that period. Today the technology for graphene synthesis is well defined and can be split into two different types of approach: top-down and bottomup. Top-down approaches involve breaking apart the stacked layers of graphite to yield single graphene sheets (micromechanical cleavage, electrochemical exfoliation, exfoliation of graphite intercalation compounds, solvent-based exfoliation, unzipping carbon nanotubes). Bottom-up methods involve synthesis of graphene from alternative carbon containing sources (epitaxial growth on silicon carbide, chemical vapor deposition on metals, substrate-free 
methods, graphene synthesis via the flash pyrolysis of the solvothermal product of sodium and ethanol, etc.) $[10,12]$. In order for graphene's potential to be fully realized and to satisfy requirements of the endapplication convenient methods for its synthesis should be developed.

Epitaxial growth of graphene on silicon carbide single crystals (4H-SiC or $6 \mathrm{H}-\mathrm{SiC}$ polytypes) is one of the highly popular techniques. Formation of graphene on silicon carbide $(\mathrm{SiC})$ proceeds via the preferential sublimation of silicon from the $\mathrm{SiC}$ surface and subsequent graphitization of the excess carbon atoms left behind [13]. This process occurs at high temperatures $\left(>1000{ }^{\circ} \mathrm{C}\right)$ and is generally performed in ultra-high vacuum (UHV) conditions, while growth in argon atmospheres [14] or at presence of small quantities of disilane [15] is also used. Of course, expensive singlecrystal $\mathrm{SiC}$ substrates of large diameter are required to obtain graphene of good quality. However, an approach that uses polycrystalline or amorphous $\mathrm{SiC}$ for synthesis of graphene micro- and nanocomposites could be used as an alternative technique $[16,17]$.The advantage of this approach is the large-scale production of graphene at low cost.

The aim of our work is to test the possibility to obtain weakly coupled or free-standing graphene sheets of acceptable quality using thermal decomposition process of $\mathrm{SiC}$ powder with spatial arrangement of $\mathrm{SiC}$ crystals in the crucible that provides sublimation almost from all their surfaces. We suggest the use of polycrystalline silicon carbide to produce both nanocomposite and graphene sheets of millimeter size. This approach is intermediate between the expensive technology where industrial substrates are used and cheap technology for producing graphene nanocomposite using micro- and nanocrystals of $\mathrm{SiC}$. We have shown that processing conditions providing intensive decomposition from all facets of the large-size $\mathrm{SiC}$ powder crystals can get a significant yield of graphene plates sized up to few millimeters.

\section{Experimental}

The coarse-grained $\mathrm{SiC}$ powder $(0.1 \ldots 2 \mathrm{~mm})$ of $6 \mathrm{H}$ polytype was preliminary synthesized by direct processing of pure carbon and silicon powders. The oven REDMET-30 with resistive heater was employed. Most of crystals were faceted and have a typical habitus for this polytype (Fig. 1a). At the next stage the $\mathrm{SiC}$ decomposition and synthesis of carbon-graphene composite were performed. The crucible heated up to $2500{ }^{\circ} \mathrm{C}$ providing the intensive sublimation of $\mathrm{SiC}$. Duration of processing varied from 10 up to 15 hours. In contrast to general layout in the crucible for large single crystals (Fig. 2), proposed in the approach are coarsegrained $\mathrm{SiC}$ crystallites placed along the walls of the graphite crucible. This arrangement allows for evaporation from almost all sides of each crystallite.
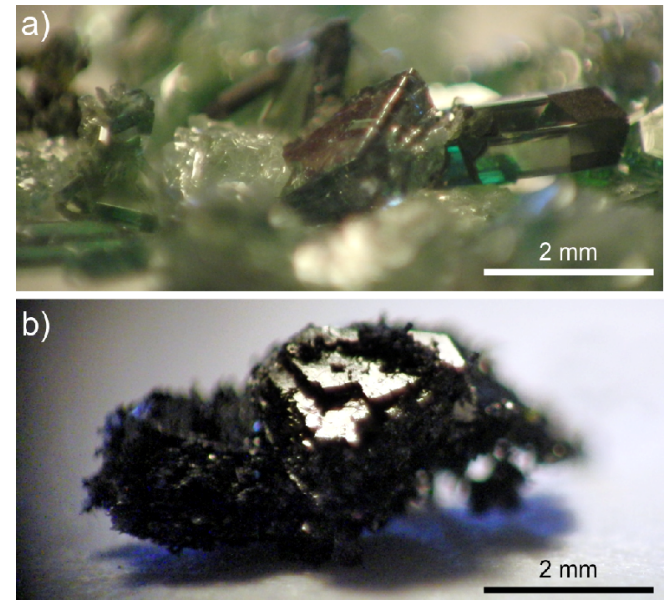

Fig. 1. Macro-scale image of initial crystalline SiC "powder" $(a)$ and carbon-graphene composite after thermal processing $(b)$.

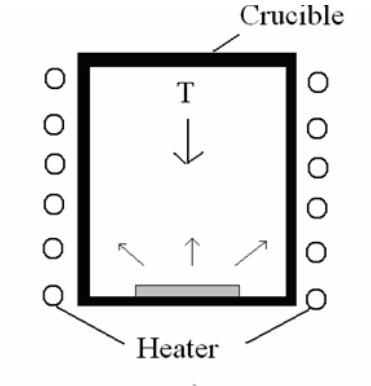

(a)

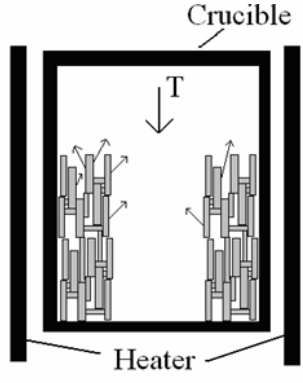

(b)
Fig. 2. Layout of $\mathrm{SiC}$ crystals in the crucible: traditional method for providing graphene layer synthesis on one (free) surface of crystal $(a)$; the proposed method providing synthesis of graphene on arbitrary facets of crystals $(b)$.

The structure and composition of the synthesized material were investigated using optical, scanning electron (SEM) and atomic force microscopy (AFM) as well as Micro-Raman spectroscopy. Micro-Raman spectra were measured in backscattering geometry using the triple Raman spectrometer T-64000 Horiba JobinYvon equipped with a cooled CCD detector. Raman spectra were measured at room temperature using $488.0 \mathrm{~nm}$ line of $\mathrm{Ar}^{+} / \mathrm{Kr}^{+}$ion laser for excitation. Excited radiation was focused on the sample surface with $100 \times$ optical objective. The laser power on the sample surface was less than $1 \mathrm{~mW}$. Raman spectra of mechanically exfoliated onto $\mathrm{SiO}_{2}$ substrate single-layer graphene sample were measured for reference.

\section{Results and discussion}

The synthesized material is composed from black powder and plates with a smooth shiny surface (Fig. 1b). The size of these plates can reach up to a few millimeters. A priori, we assumed that these plates are 
graphene-containing phase and selected them for further structural investigations. Extraction of plates from compound produced by complete thermal decomposition of $\mathrm{SiC}$ powder is a simple procedure in our case. They can be easily separated mechanically, for instance. Enlarged SEM images of small and large pieces of the composite are shown in Fig. 3.

Correlational AFM and Raman studies of the structure and phase composition performed on the plates of large sizes. As an example, in Fig. $3 \mathrm{~b}$ circle marked areas where appropriate measurement were carried out. AFM image of plate surface in the marked area is shown in Fig. 3c. Presence of growth terraces with the width from tens of nanometers up to $1 \mu \mathrm{m}$ is typical for the surface of plates. There are equally smooth, uniform surface areas as well as areas with significant defects (marked by arrows). SiC substrate roughening as the graphene forms is the major problem with UHV grown Si-face epitaxial graphene production [12]. The slower rate of $\mathrm{Si}$ removal from the interface on the Si-face of $\mathrm{SiC}$ would also explain the higher surface roughness after graphitization. It is known from studies of $\mathrm{SiC}$ growth that on the $\mathrm{Si}$-face excess $\mathrm{Si}$ nucleates on the $\mathrm{SiC}$ terraces and gives rise to faceting and twinning [18]. Si trapped at the graphene/ $\mathrm{SiC}$ interface would recrystallize as $\mathrm{SiC}$ clusters on the $\mathrm{SiC}$ terraces that would then nucleate islands and ultimately cause the surface to roughen. Taking into account this feature of graphenelike layers formation, it could be assumed that rough and smooth fragments of surfaces studied correspond to $\mathrm{Si}$ or $\mathrm{C}$-surface of crystals in the initial $\mathrm{SiC}$ powder.
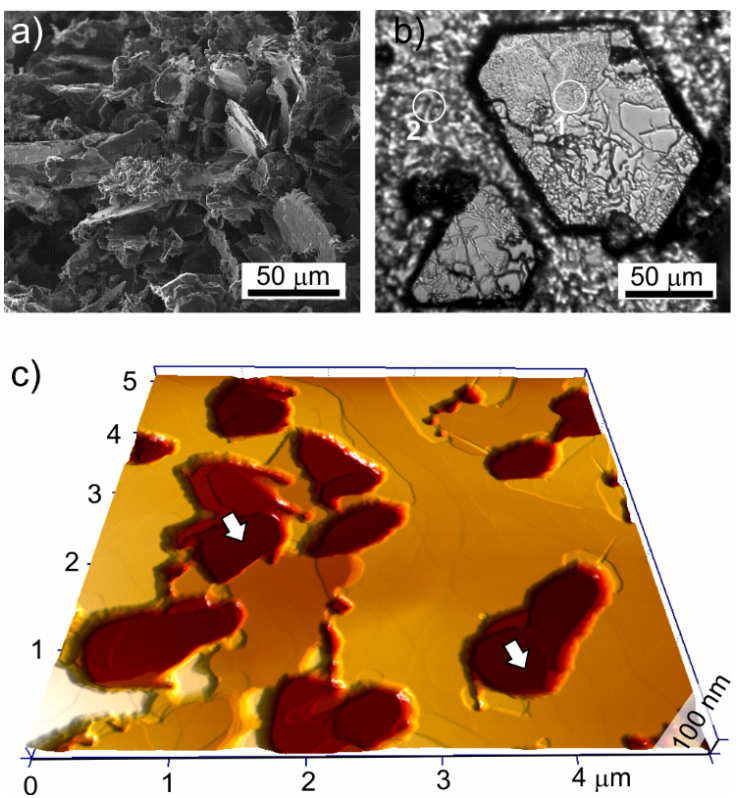

Fig. 3. SEM image of carbon-graphene composite synthesized from $\mathrm{SiC}$ powder $(a)$, image of the extracted smooth plates of graphene-containing phase translated to the flat substrate $(b)$. Circles 1 and 2 denote Raman measurements areas. 3D AFM image of surface in the circle $1(c)$.
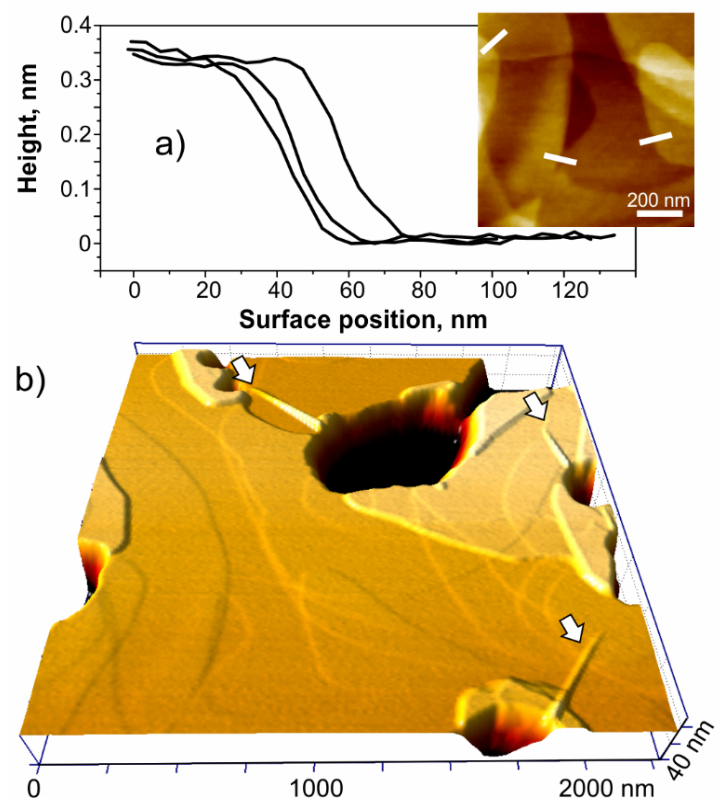

Fig. 4. The height profiles of surface terraces $(a)$. The insert illustrates points where measurements have been carried out. AFM image $(b)$ of terraces and ridges (arrow marked bulged fragments) of the graphene film.

More details of surface topography of the graphene-like film are presented in Fig. 4. It is seen that the height of the terraces is about $0.35 \mathrm{~nm}$. This value is consistent with the values for the interlayer spacing in turbostratic $(>0.342 \mathrm{~nm})$ and crystalline $(0.335 \mathrm{~nm})$ graphene [10]. In addition, bright stripes across terraces are clearly visible (indicated by arrows in Fig. 4b). They usually start at the edges of large defects. These features are consistent with the ridges and wrinkles reported in earlier studies [19], which are usually observed on graphene grown on $\mathrm{SiC}$ surface [16] due to the large difference in thermal expansion coefficient between epitaxial graphene layers and $\mathrm{SiC}$.

Fig. 5 shows typical Raman spectra of the investigated samples measured in various areas of the investigated samples, and the reference spectrum of mechanically exfoliated single-layer graphene. As can be seen, the measured Raman spectra demonstrate appearance of the narrow $G$ band at approximately $1582 \mathrm{~cm}^{-1}$, which is indicative of the hexagonal graphene lattice formation, and good expressed 2D band at $\sim 2720 \mathrm{~cm}^{-1}$ that originates from a double electronphonon resonance (DR) Raman process [20]. Small halfwidth of the $\mathrm{G}$ bands $\left(\sim 14 \mathrm{~cm}^{-1}\right)$ indicates good crystal quality of the investigated graphene layers. In addition, the low relative intensity of defect-activated D band in all Raman spectra indicates small amount of defects in the investigated graphene samples. In contrast to the $\mathrm{G}$ band, position and shape of 2D band significantly varies depending on the measured area of the samples. As can 


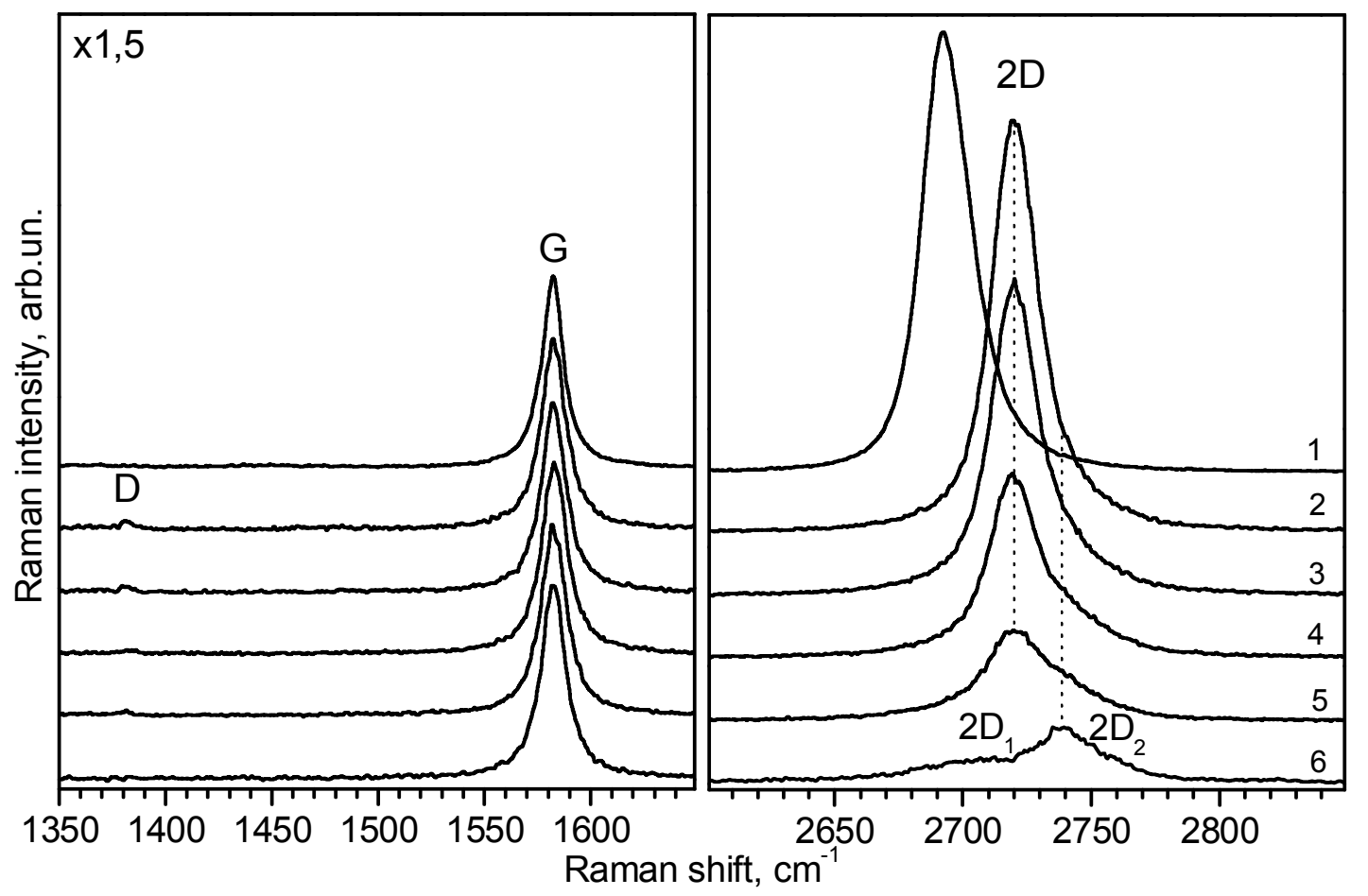

Fig. 5. Raman spectra measured at various areas of the sample shown in Fig. $2 b$. The curves 2 to 4 were measured within the circle 1 and curves 5 to 6 within the circle 2 . The Raman spectrum for mechanically exfoliated graphene is shown for reference (curve 1).The spectra are normalized to the intensity of the G-band, and spectra on left panel are multiplied by the factor 1.5 for convenience reasons.

be seen from Fig. 4, 2D band in the spectrum 2 is a single peak at $2720 \mathrm{~cm}^{-1}$. However, noticeable highfrequency asymmetry of the $2 \mathrm{D}$ band appears in the spectra 3 to 5 . Finally, the spectrum 6 demonstrates a clear expressed doublet structure of the 2D band with $2 \mathrm{D}_{1}$ and $2 \mathrm{D}_{2}$ components at 2700 and $2740 \mathrm{~cm}^{-1}$, correspondingly.

The Raman spectrum 2 (Fig. 5), in general, is similar to the spectrum of mechanically exfoliated single-layer graphene (spectrum 1) with the 2D band having homogeneous single-component contour with the half-width close to $21 \mathrm{~cm}^{-1}$ and intensity much higher than that of the $\mathrm{G}$ band. The intensity ratio $\mathrm{I}_{2 \mathrm{D}} / \mathrm{I}_{\mathrm{G}}$ for the spectrum 2 is 3.5 , which is close to the corresponding value for single-layer graphene (in our case 3.7). However the position of the 2D band in the spectrum 2 at $2720 \mathrm{~cm}^{-1}$ is significantly blue-shifted by $\sim 28 \mathrm{~cm}^{-1}$ as compared to the corresponding value of $2692 \mathrm{~cm}^{-1}$ for single-layer graphene.

One of the possible reasons for the observed blueshift of the $2 \mathrm{D}$ band could be residual biaxial compressive strains in the investigated layers, which is typical situation for the thermally-induced graphene layers grown on $\mathrm{SiC}$ substrates [21]. However, the strain-induced shift of the 2D band by $\sim 30 \mathrm{~cm}^{-1}$ should be accompanied by the corresponding high-frequency shift of the $G$ band by $\sim 8 \mathrm{~cm}^{-1}$, which is not the case [22]. A slight high-frequency shift of the $G$ and $2 D$ bands can also be caused by the uncontrolled doping of the graphene layers in the growth process, but in this case we should expect a shift of the G and 2D bands of the same order [23].

The nature of such unexpectedly high blue-shift of the $2 \mathrm{D}$ band at almost the same position of the $\mathrm{G}$ band in our opinion could be understood, if consider the peculiarities of the investigated graphene multilayer structures, and in particular their stacking order. Thus, an alternative mechanism of the 2D band high-frequency shift at invariable position of the $G$ band in case of multilayer graphene structures could be explained by modification of the electron pi-bands near the K-point of the graphene Brillouin zone caused by changes in interlayer interaction of the graphene layers. In the conditions of DR process, it will lead to a shift in phonon wave vectors and corresponding shift of the $2 \mathrm{D}$ band [24]. Modification of the electronic bands in our case can be caused by rotational disorder of the 
investigated graphene layers due to peculiarities of the thermally activated growth process taking place on different facets of the initial $\mathrm{SiC}$ crystals [25].

In case of two- or multilayer graphene, modification of the electron pi-bands can be caused by decrease of coupling of graphene layers due to their rotational disorder (deviation from $\mathrm{AB}$ stacking). As it was shown theoretically from computation of electron structure [26,27], rotationally misoriented two-layer graphene preserves linear dispersion of the electron pibands near the K-point, but with a less slope (less Fermi velocity) [28]. Thus, the $2 \mathrm{D}$ band in the Raman spectrum of misoriented two-layer graphene is a single peak with the intensity higher than for the $G$ band, similarly to single-layer graphene, but shifted to higher frequencies [24, 29].

Therefore, having in mind that we deal with thick multilayer graphene structures, it is reasonable to assume that unusual blue shift of the $2 \mathrm{D}$ band in our case is caused by the peculiarities of the stacking order of the graphene layers and corresponding interaction between them [30]. It was shown earlier [31] that the nature of thermally induced grown graphene layers on Cterminated $\mathrm{SiC}$ substrate is turbostatic with rotational disorder and weak coupling between graphene layers. In contrast, on Si-terminated surfaces carbon atoms in the growth process are covalently bounded to the underline $\mathrm{Si}$ atoms leading to growth of graphene layers, which are aligned with respect to the substrate [25].

It should be noted that in the annealed samples we didn't find any evidence of the Raman signatures from the residue of the initial $\mathrm{SiC}$ crystals, which means that almost all $\mathrm{Si}$ atoms were sublimated in the annealing process from the initial SiC crystals. So, we can assume that investigated samples are composed from the weakly coupled graphene layers with the stacking order depending on the orientation of the initial $\mathrm{SiC}$ crystals. In such a way, the spectrum 2 corresponds to weakly coupled graphene multilayers apparently grown on C-terminated side of the initial $\mathrm{SiC}$ crystals. Then, the spectrum 6 would correspond to growth of graphene layers on $\mathrm{Si}$-terminated surface, which results in increased coupling between graphene layers. The observed relatively low intensity of the 2D band and appearance of two well-resolved $\left(2 \mathrm{D}_{1}\right.$ and $\left.2 \mathrm{D}_{2}\right)$ components with domination of the high-frequency $2 \mathrm{D}_{2}$ component is typical for Bernal graphite with $\mathrm{AB}$ stacking of the graphene layers [32]. The spectra 3 to 5 show transition between two cases mentioned above, which is accompanied by the decrease in intensity of the 2D band, appearance and growth in intensity of the high-frequency $2 \mathrm{D}_{2}$ component at $2740 \mathrm{~cm}^{-1}$, which, in general, reflects the increase in coupling of the graphene layers.

Finally, observation of forbidden $\mathrm{D}$ bands in the Raman spectra of the investigated graphene layers (spectra 2 to 5) with the relative intensity (compared to the $\mathrm{G}$ band) increasing simultaneously with the intensity of the 2D bands is unusual and needs additional discussion. The related with defects $\mathrm{D}$ band is forbidden in the first-order Raman scattering of bulk graphite as well as single-layer graphene and can be activated by structural defects. Processes responsible for the defectinduced $\mathrm{D}$ band and two-phonon 2D band within the DR conditions are competitive, i.e., the increase in intensity of the D band due to structural defects unlike our case should lead to decrease in intensity of the $2 \mathrm{D}$ band. Rotational disorder of the graphene layers was also shown to lead to activation of forbidden defect-bound D bands in the Raman spectra due to perturbation caused by decrease in interlayer interaction $[33,34]$. This phenomena was registered in the Raman spectra of the graphene grown by chemical deposition [35] and by thermal decomposition from the solid phase [36]. The observed decrease in intensity of the $\mathrm{D}$ band for the spectra 2 to 6 within the proposed model can be caused by increase in interaction between the graphene layers, which confirms the above assumption. Complete disappearance of the $\mathrm{D}$ feature in the spectrum 6 is additional argument for the defect-free $\mathrm{AB}$ stacked graphite in this case.

\section{Conclusions}

We report the growth of high-quality and millimeterssized substrate-free graphene multilayers with the interlayer coupling depending on the geometry of the thermal decomposition process and orientation of the initial SiC crystals. With free arrangement of crystallites along the walls of crucible the sublimation from their entire surfaces is possible. The decomposition process intensity is so high that no $\mathrm{SiC}$ phase was detected in synthesized carbon-graphene composite. This simple method provides a promising approach for industrial fabrication of substrate-free weakly coupled graphene sheets from a coarse-grained $\mathrm{SiC}$ powder.

Carbon rest consists from mixture of graphene flakes and spongy carbon. In summary, this method is feasible for the production of free-standing single-layer graphene, which is expected to promote further development of other graphene-based technologies such as the manufacturing electrodes for ionistors, batteries, and hydrogen-storage materials.

\section{Acknowledgements}

The work was supported in part by the Science and Technology Center in Ukraine (STCU) and National Academy of Sciences of Ukraine within the framework of the Targeted Research \& Development Initiatives (TRDI) Program under the Grant Project \#5716 "Development of Graphene Technologies and Investigation of Graphene-based Nanostructures for Nanoelectronics and Optoelectronics" (2012-2014). Partial support by FP7-PEOPLE-2010-IRSES and Alexander von Humboldt Institutional Partnership project (DEU/1053880) are acknowledged. 


\section{References}

1. K.S. Novoselov, A.K. Geim, S.V. Morozov, D. Jiang, M.I. Katsnelson, I.V. Grigorieva, S.V. Dubonos, A.A. Firsov, Two-dimensional gas of massless Dirac fermions in graphene // Nature, 438 (7065), p. 197 (2005).

2. Y. Zhang, Y.-W. Tan, H.L. Stormer, P. Kim, Experimental observation of the quantum Hall effect and Berry's phase in graphene // Nature, 438 (7065), p. 201 (2005).

3. K.S. Novoselov, E. McCann, S.V. Morozov, V.I. Fal'ko, M.I. Katsnelson, U. Zeitler, D. Jiang, F. Schedin, A. Geim, Unconventional quantum Hall effect and Berry's phase of $2 \pi$ in bilayer graphene // Nature Physics, 2(3), p. 177 (2006).

4. K.S. Novoselov, Z. Jiang, Y. Zhang, S.V. Morozov, H.L. Stormer, U. Zeitler, J.C. Maan, G.S. Boebinger, P. Kim, A.K. Geim, Roomtemperature quantum Hall effect in graphene // Science, 315 (5817), p. 1379 (2007).

5. F. Schedin, A.K. Geim, S.V. Morozov, E.W. Hill, P. Blake, M.I. Katsnelson, K.S. Novoselov, Detection of individual gas molecules adsorbed on graphene // Nature Materials, 6(9), p. 652 (2007).

6. B. Trauzettel, D.V. Bulaev, D. Loss, G. Burkard, Spin qubits in graphene quantum dots // Nature Physics, 3(3), p. 192 (2007).

7. T. Yokoyama, Controllable spin transport in ferromagnetic graphene junctions // Phys. Rev. B, 77(7), 073413 (2008).

8. F. Rana, Graphene terahertz plasmon oscillators // Nanotechnology, IEEE Trans. 7(1), p. 91 (2008).

9. A.K. Geim, K.S. Novoselov, The rise of graphene // Nature Materials, 6(3), p. 183 (2007).

10. R.S. Edwards, K.S. Coleman, Graphene synthesis: relationship to applications // Nanoscale, 5(1), p. 38 (2013).

11. B. Lang, A LEED study of the deposition of carbon on platinum crystal surfaces // Surf. Sci. 53(1), p. 317 (1975).

12. W. Choi, I. Lahiri, R. Seelaboyina, Y.S. Kang, Synthesis of graphene and its applications: A Review // Critical Reviews in Solid State and Materials Sciences, 35(1), p. 52 (2010).

13. P. Sutter, Epitaxial graphene: How silicon leaves the scene // Nature Materials, 8(3), p. 171 (2009).

14. K.V. Emtsev, A. Bostwick, K. Horn, J. Jobst, G.L. Kellogg, L. Ley, J.L. McChesney, T. Ohta, S.A. Reshanov, J. Röhrl, Towards wafer-size graphene layers by atmospheric pressure graphitization of silicon carbide // Nature Materials, 8(3), p. 203 (2009).

15. R.M. Tromp, J.B. Hannon, Thermodynamics and kinetics of graphene growth on SiC (0001) // Phys. Rev. Lett. 102(10), 106104 (2009).

16. K. Zhu, L. Guo, J. Lin, W. Hao, J. Shang, Y. Jia, L. Chen, S. Jin, W. Wang, X. Chen, Graphene covered $\mathrm{SiC}$ powder as advanced photocatalytic material // Appl. Phys. Lett. 100(2), 023113 (2012).

17. T. Peng, H. Lv, D. He, M. Pan, S. Mu, Direct transformation of amorphous silicon carbide into graphene under low temperature and ambient pressure // Scientific Reports, 3 (2013).

18. J. Hass, W.A. De Heer, E.H. Conrad, The growth and morphology of epitaxial multilayer graphene // J. Phys.: Condens. Matter, 20(32), 323202 (2008).

19. S. Mikhailov, Physics and Applications of Graphene-Experiments. InTech, 2011.

20. L.M. Malard, M.A. Pimenta, G. Dresselhaus, M.S. Dresselhaus, Raman spectroscopy in graphene // Phys. Repts. 473, p. 51 (2009).

21. Z. H. Ni, W. Chen, X. F. Fan, J. L. Kuo, T. Yu, A. T. S. Wee, Z. X. Shen, Raman spectroscopy of epitaxial graphene on a SiC substrate // Phys. Rev. $B, 77,115416$ (2008).

22. T.M.G. Mohiuddin, A. Lombardo, R.R. Nair, A. Bonetti, G. Savini, R. Jalil, N. Bonini, D.M. Basko, C. Galiotis, N. Marzari, K.S. Novoselov, A.K. Geim, and A.C. Ferrari, Uniaxial strain in graphene by Raman spectroscopy: $\mathrm{G}$ peak splitting, Grüneisen parameters, and sample orientation // Phys. Rev. B, 79, 205433 (2009).

23. C. Casiraghi, S. Pisana, K.S. Novoselov, A.K. Geim, A.C. Ferrari, Raman fingerprint of charged impurities in graphene // Appl. Phys. Lett. 91, 233108 (2007).

24. P. Poncharal, A. Ayari, T. Michel, and J.-L. Sauvajol, Raman spectra of misoriented bilayer graphene // Phys. Rev. B, 78, 113407 (2008).

25. J. Hass, W.A. de Heer and E.H. Conrad, The growth and morphology of epitaxial multilayer graphene // J. Phys.: Condens. Matter, 20, 323202 (2008).

26. S. Latil, V. Meunier, L. Henrard, Massless fermions in multilayer graphitic systems with misoriented layers: $\mathrm{Ab}$ initio calculations and experimental fingerprints // Phys. Rev. B, 76, 201402 (2007).

27. J.M.B. Lopes dos Santos, N.M.R. Peres, and A.H. Castro Neto, Graphene bilayer with a twist: Electronic structure // Phys. Rev. Lett. 99, 256802 (2007).

28. Yuehua $\mathrm{Xu}$, Xiaowei $\mathrm{Li}$ and Jinming Dong, Infrared and Raman spectra of AA-stacking bilayer graphene // Nanotechnology, 21, 065711 (2010).

29. Z. Ni, Y. Wang, T. Yu, Y. You, Z. Shen, Reduction of Fermi velocity in folded graphene observed by resonance Raman spectroscopy // Phys. Rev. B, 77, 235403 (2008).

30. C. Faugeras, A. Nerrière, M. Potemski, A. Mahmood, E. Dujardin, C. Berger and W.A. de Heer, Few-layer graphene on $\mathrm{SiC}$, pyrolitic graphite, and graphene: A Raman scattering study // Appl. Phys. Lett. 92, 011914 (2008).

31. K.V. Emtsev, F. Speck, Th. Seyller, and L. Ley, Interaction, growth, and ordering of epitaxial 
graphene on $\mathrm{SiC}\{0001\}$ surfaces: A comparative photoelectron spectroscopy study // Phys. Rev. B, 77, 155303 (2008).

32. H. Wilhelm, M. Lelaurain, E. McRae, and B. Humbert, Raman spectroscopic studies on welldefined carbonaceous materials of strong twodimensional character. J. Appl. Phys. 84,6552 (1998).

33. R. Rao, R. Podila, R. Tsuchikawa, J. Katoch, D. Tishler, A.M. Rao, M. Ishigami, Effects of layer stacking on the combination Raman modes in graphene // ACS Nano, 5(3), p. 1594 (2011).

34. A.K. Gupta, Youjian Tang, V.H. Crespi, and P.C. Eklund, Nondispersive Raman D band activated by well-ordered interlayer interactions in rotationally stacked bilayer graphene // Phys. Rev. $B, 82,241406(\mathrm{R})$ (2010).

35. M. Okano, R. Matsunaga, K. Matsuda, S. Masubuchi, T. Machida, Y. Kanemitsu, Raman study on the interlayer interactions and the band structure of bilayer graphene synthesized by alcohol chemical vapor deposition // Appl. Phys. Lett. 99, 151916 (2011).

36. J. Hofrichter, B.N. Szafranek, M. Otto, T.J. Echtermeyer, M. Baus, A. Majerus, V. Geringer, M. Ramsteiner, H. Kurz, Synthesis of graphene on silicon dioxide by a solid carbon source // Nano Lett. 10, p. 36 (2010). 